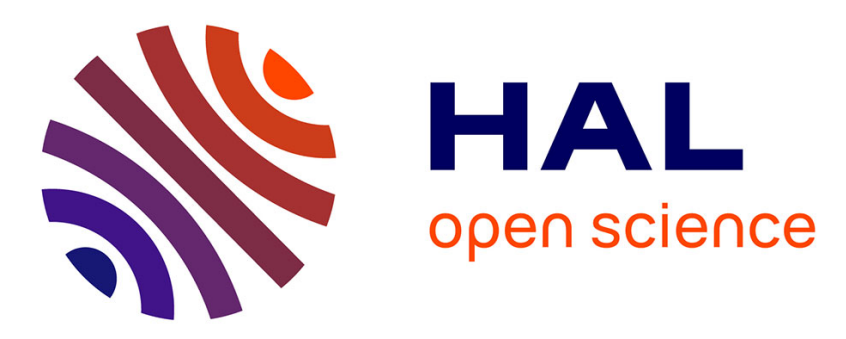

\title{
Drag in wet granular materials
}

Riccardo Artoni, Giovanni Loro, Patrick Richard, Fabio Gabrieli, Andrea Santomaso

\section{To cite this version:}

Riccardo Artoni, Giovanni Loro, Patrick Richard, Fabio Gabrieli, Andrea Santomaso. Drag in wet granular materials. Powder Technology, 2019, 356, pp. 231-239. 10.1016/j.powtec.2019.08.016 . hal-02278243

\section{HAL Id: hal-02278243 \\ https://hal.science/hal-02278243}

Submitted on 4 Sep 2019

HAL is a multi-disciplinary open access archive for the deposit and dissemination of scientific research documents, whether they are published or not. The documents may come from teaching and research institutions in France or abroad, or from public or private research centers.
L'archive ouverte pluridisciplinaire HAL, est destinée au dépôt et à la diffusion de documents scientifiques de niveau recherche, publiés ou non, émanant des établissements d'enseignement et de recherche français ou étrangers, des laboratoires publics ou privés. 


\title{
Drag in wet granular materials
}

\author{
Riccardo Artoni, Giovanni Loro, Patrick Richard \\ IFSTTAR, MAST, GPEM, F-44344 Bouguenais, France
}

Fabio Gabrieli

GeoTechLab, Department of Civil, Architectural and Environmental Engineering, University of Padova, Via Ognissanti 39, 35129 Padova, Italy

Andrea C. Santomaso

APTLab, Advanced Particle Technology Laboratory, Department of Industrial Engineering, University of Padova, Via Marzolo 9, 35131 Padova, Italy

\begin{abstract}
We have studied experimentally the effect of the water content on the drag force applied by a wet granular system on a probe moving through it at constant velocity. Similar to what has been observed for dry granular systems, the drag force is found to be independent of the velocity of the probe. By comparing our result with the dry case, we have shown that three main physical mechanisms occur: cohesion (consequence of capillary forces), contact lubrication and dilatancy induced by cohesion. The influence of the latter mechanism prevails in our system and the relative influence of the two former ones is found to depend on grains' characteristics. At important water contents, the granular system may become heterogeneous and its structure is modified by the motion of the probe. Our analysis is strengthened by shear cell experiments which also point out the importance of the geometry of the system on the effect of the water content.
\end{abstract}

Keywords: wet granular material, drag, mixing, shear testing

\section{Introduction}

The study of forces induced on bodies immersed in granular media has several applications e.g. mixing of granular materials and pastes, predicting damage induced by landslides on structures and designing retaining or dissipative systems for geohazards. In all the cases, there is a strong need for the development of (1) conceptual models and (2) new sensors for monitoring and control. In this perspective, the measurement of the drag force exerted by the material on a moving intruder (whose size is much larger than the particle diameter) can be seen as an opportunity in order to gather information about the rheology of granular materials and their interaction with structures. For these reasons, in the last decades there has been interest on characterizing the drag force on objects in dense granular media.

Wieghardt $(1974,1975)$ was one of the first to study "granular" drag. His experiments consisted in moving partially immersed rods through sand beds. The drag force is found to be dependent on both the geometry

Preprint submitted to Powder Technology of the rod and its velocity although the latter parameter has to be relatively large to have a significant effect. The behavior at low velocity can be understood by assuming that the interactions between the rod and the surrounding grains are mostly governed by sliding friction, a velocity independent force. In contrast, collisions between the rod and the surrounding grains lead to a velocity dependent dissipation. They are no more negligible at important velocities and can explain the observed weak influence of the velocity.

A large amount of work in this area has been performed by Albert and coworkers (Albert et al., 1999, 2001a,b). In particular, they first investigated the effect of velocity, particle size, object size and depth on the resistance provided by dense granular materials on a partially immersed, slowly moving cylinder (Albert et al., 1999). The symmetry axis of the cylinder was vertically oriented and the motion was horizontal. Subsequently, they focused on the effect of the shape of the dragged object on the drag force and the relevant scalings (Al- 
bert et al., 2001a). The main results which were obtained in these works are that the drag is independent of velocity (in the quasistatic and dense flow regimes), and particle diameter (at least as soon as the intruder size is large with respect to the particle diameter), but depends on the depth of the object by a scaling which depends on object shape. Moreover, the drag force appears to increase linearly with the object size. These results stem from the frictional nature of granular materials, in which the yield stress is proportional to pressure. In a further work (Albert et al., 2001b), they also characterized drag force fluctuations, which they linked to jamming events occurring in the bulk of the material.

Later, Chehata et al. (2003) studied the drag force acting on a cylinder immersed in a dense, quasi twodimensional vertical chute flow. They varied the flow rate, the object diameter and particle size, finding a rateindependent behavior, a linear dependence on cylinder size and a weak dependence on particle size. By means of 2D numerical simulations, Wassgren et al. (2003) studied the flow of a dilute granular system around a disk. They found that the drag coefficient decreases with decreasing normal coefficient of restitution and is relatively insensitive to the friction coefficient. Interestingly they also reported shock and expansion fan structures, similar to those observed in gas dynamics. Seguin et al. (2011) coupled drag force measurements with Particle Image Velocimetry (PIV) to characterize the case of a horizontal cylinder moving into a granular medium. The drag force behavior is in agreement with previous works, apart from a nonlinear dependence of the force on particle size, which seems to be present when the latter is below $1 \mathrm{~mm}$. They compared the experimental velocity profiles around the object with predictions from an extended kinetic theory. In a different configuration, a rod rotating at a given depth in a box filled with grains, Guillard et al. (2013) obtained that the torque needed for stirring the material increased at the beginning, then dropped down, eventually reaching a stationary value which appeared to be independent on the depth. Clearly, the scaling on depth is completely different from the literature referenced above, but may be ascribed to the peculiar geometry used by the authors. More recently, Kumar et al. (2017) numerically studied the scaling of the drag force on velocity. They extended previous studies by including flows well beyond the slow quasistatic regime, and identified the existence of three regimes (rate-independent, linear and quadratic dependence of the drag force on velocity).

All the works referenced above have dealt with dry, cohesionless granular materials. Rheology of partially wet conditions have been discussed with reference to rheometer experiments on plastic beads (Badetti et al., 2018), rapid shear cell tests with glass beads (Liao and Hsiau, 2010), quasistatic tests in a Schulze shear cell with glass beads (Louati et al., 2015, 2017) and column collapse tests with glass beads (Artoni et al., 2013; Gabrieli et al., 2013) and different types of sand (Santomaso et al., 2018). Collet et al. (2010, 2011) also studied the effect of liquid addition during mixing of fine powders and glass beads by analysing the electric current absorbed by the mixer.

An analogous approach in the pharmaceutical field was initially proposed by Leuenberger (1982) for high shear wet granulation experiments. Leuenberger linked the motor power consumption profiles to the level of liquid saturation of the wet agglomerate and proposed a criterion for determining the amount of liquid required for granulating. Power consumption is also used to monitor and control the evolution of wet mixing in other application fields, such as concrete production (Cazacliu and Roquet, 2009). However, power-consumption has a number of drawbacks (current drift with motor temperature, lag between current and voltage, use of over-sized motors and gearmotors which hinder current variations) that makes direct force or mixer torque measurements a reliable alternative for monitoring wet mixing processes (Jørgensen et al., 2004; Cazacliu, 2008; Cavinato et al., 2010; Chitu et al., 2011). The study of the force required to move an intruder through a wet granular mixture has therefore important industrial applications. Torque is a measure of the force needed to rotate the impeller which depends on the resistance of the wet mass against rotation of the impeller blades (Bouwman et al., 2005). Experiments monitoring the torque in high shear mixers therefore share some similiarity with the present case of the drag force applied by a wet granular system on a probe moving through it at constant velocity. The main difference however is the intensity of mixing, which is much larger in the high shear mixers, with significant centripetal accelerations of the wet material able to generate a toroidal flow pattern (Cavinato et al., 2013) not present in the our pan mixer.

The behavior of wet granular materials can be characterized by a dimensionless number (Bond number), which compares body to capillary forces, and provides the framework to understand why increasing the particle size or decreasing the fluid surface tension yield a mechanical response similar to dry conditions. Bond number is defined as $B o=\rho_{p} g d_{p}^{2} / \gamma$, where $\rho_{p}$ is particle density, $d_{p}$ a characteristic particle diameter, $\gamma$ the surface tension of the liquid and $g$ the gravitational acceleration. Particle shape also plays an important role: 
for angular particles, capillary bridges, responsible of the attraction between grains, spread on the grain surface and are partially captured by surface roughness.

When focusing on the drag force on an object moving through a granular material, one may imagine that, if cohesion is introduced into the system, for example by wetting the grains, a cohesive stress scale is introduced which may change the drag scaling. It is important to stress that the addition of a liquid not only gives a tensile strength to the material, but also lubricates contacts, which results in a lower macroscopic coefficient of friction (Santomaso et al., 2018). It is therefore interesting to study the effect of the addition of liquid (in this case, water) to the drag force on an intruder moving through a granular medium. This is the subject of the present work, which is, to our knowledge, the first study on drag forces on objects moving through wet granular media. It has to be emphasized that the experiments discussed in the paper were performed at a large, industrial scale, in the framework of a global project on the efficiency of industrial mixing of granular materials.

In the following, Section 2 presents the equipment, materials and procedures used; in Section 3 the raw results are briefly discussed, while Section 4 deals with an interpretation of the drag results by renormalization and comparison with shear cell analyses. Section 5 summarizes the main results and concludes the paper.

\section{Materials \& Methods}

\subsection{Materials}

Experiments were performed on two sands, Fontainebleau sand (FS) and Teillé sand (TS) (Fig. 1). Fontainebleau sand is a fine siliceous sand with a quite narrow PSD. Teillé sand is a coarser and more heterogeneous river sand. Particle size distributions (PSD) for the two materials were determined by sieving and are displayed in Fig. 1. Material parameters are also summarized in Tab. 1. Particle shape was evaluated by computer-assisted microscopy using Fiji software (Schindelin et al., 2012). The two sands resulted made of rounded particles with similar circularity (evaluated as $\sqrt{4 \pi A / P^{2}}$, where $A$ is the projected area and $P$ the perimeter). Before the tests, materials were air dried to reach a sufficiently low initial moisture level. The wetting liquid added in the subsequent phases was simply tap water.

\subsection{Mixer}

In this work, due to the need of mixing a certain amount of fluid with the granular medium, drag measurements were performed within a modified mixer.
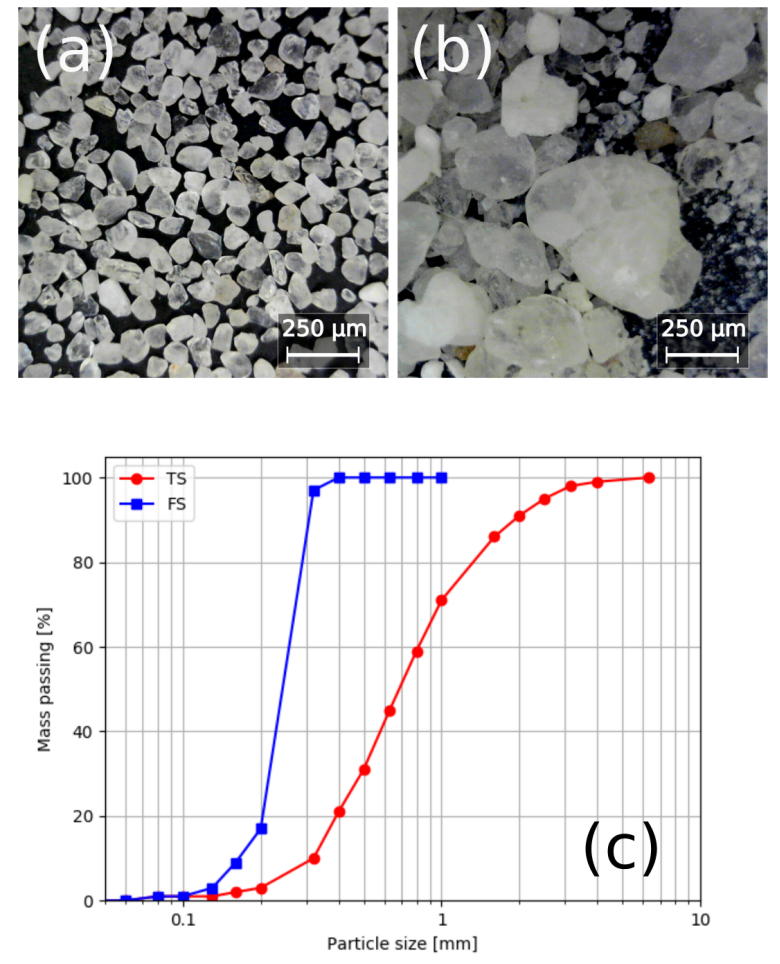

Figure 1: Microscope pictures of (a) Fontainebleau and (b) Teillé sand. (c) The cumulative particle size distributions of the two types of sand show that the former is coarser and its size distribution broader.

\begin{tabular}{cccc}
$\begin{array}{c}\text { Sand } \\
\text { reference }\end{array}$ & $\begin{array}{c}d_{50} \\
(\mu \mathrm{m})\end{array}$ & $\begin{array}{c}\text { Particle density } \\
\left(\mathrm{kg} / \mathrm{m}^{3}\right)\end{array}$ & $\begin{array}{c}\text { Circularity } \\
(-)\end{array}$ \\
\hline Teillé (TS) & 690 & 2633 & $0.86 \pm 0.05$ \\
Fontainebleau (FS) & 250 & 2643 & $0.87 \pm 0.04$
\end{tabular}

Table 1: Characteristic properties of the sands used in this work.

This was originally a tractor-mounted $1 \mathrm{~m}^{3}$ pan mixer, characterized by 5 blades positioned at different distances from the central axis, and rotating in an annular space (see Figure 2). In our case, the mixer was connected to an asynchronous motor equipped with an inverter to easily vary the velocity. One of the blades was replaced by the force measurement system (see Section 2.3). With regard to the dimensions of the mixer, the inner and outer diameter of the mixing zone are respectively $500 \mathrm{~mm}$ and $1820 \mathrm{~mm}$, and the maximum height attainable by the material from the bottom of the vessel is $450 \mathrm{~mm}$. The angular velocity of the blades was varied in the range $\omega=2.1-21 \mathrm{rpm}$. 


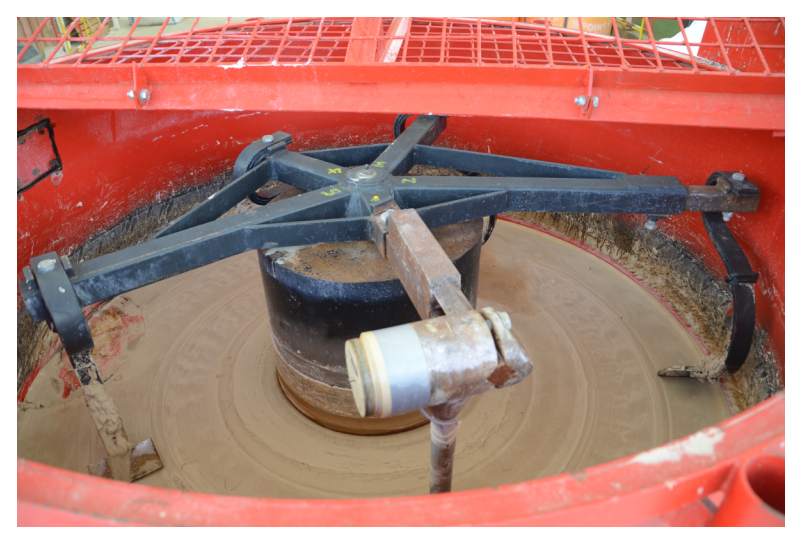

Figure 2: Internal view of the mixer adapted for this study. The four blades placed at different radial positions allow to mix the sand and the water. The upper part of the force measurement system is visible in the foreground.

\subsection{Force measurement technique}

In order to evaluate the drag induced on a body moving through a granular medium, one of the blades of the mixer was replaced by a shaft at the end of which a spherical body was attached. The shaft was made of aluminium and had the length and diameter of $280 \mathrm{~mm}$ and $35 \mathrm{~mm}$; the sphere (steel) had the two horizontal poles flattened, and had a diameter of $100 \mathrm{~mm}$ and an effective height of $90 \mathrm{~mm}$. The probe axis was mounted at a distance of $620 \mathrm{~mm}$ from the center of rotation of the blades (which yields a velocity range of 0.14 to $1.4 \mathrm{~m} / \mathrm{s}$ ). The height of the sphere with respect to the bottom of the mixer was kept constant, and corresponded to $50 \mathrm{~mm}$ (from the bottom part of the sphere), which was chosen based on a balance between the need of maximizing the highest possible level over the sphere and that of minimizing the interaction with the bottom boundary. The shaft was equipped with two pairs of strain gauges glued to its upper part. They allowed to monitor the bending of the shaft in both the streamwise and the transverse direction. The strain gauges were placed $230 \mathrm{~mm}$ above the sphere. The acquisition of the signals was performed within the mixer in a box located above the shaft which contained two conditioning cards (18bit Mantracourt DSCUSB OEM), a lightweight single board computer (Raspberry Pi Model 3) for data acquisition, and a battery for power supply. The force measurement system was therefore autonomous. Basic control of the acquisition procedure and data transfer were performed by means of an external laptop connected through a wireless link. Calibration was made by means of known masses attached to the sensing body. It is important to point out that the measurement set-up allows to quantify the moment acting on the shaft, not the force. Given that the material induces a distributed load on the sphere-and-shaft system which profile is not known a priori, it is not possible to deduce the total drag force rigorously from the bending moment. However, even if it is specific to the setup, this quantity can be used to investigate the relative effect of parameters such as velocity, material amount and water addition on the resistance provided by the granular medium on the motion of the intruder. For each configuration, the average moment was obtained by averaging the measured moment in time, while the standard deviation of the signal was taken as a measure of the uncertainty.

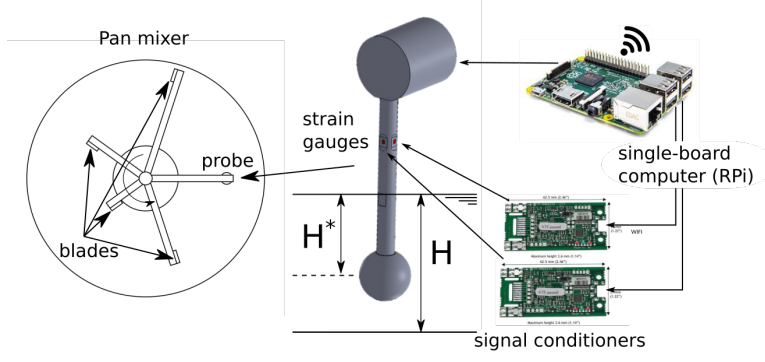

Figure 3: Top-view sketch of the mixer configuration with the probe location and detail of the system used for measuring the drag induced by the granular material. The heights $H$ and $H^{*}$ are respectively the position of the free surface with respect to the the bottom of the mixer and the level above the half-sphere plane $H^{*}$.

\subsection{Level measurement}

The level of material in the mixer, $H$, was determined after mixing by means of a rigid ruler placed $10 \mathrm{~cm}$ downstream the probe. This was chosen due to the need of measuring the level near the probe. The uncertainty estimation for this measurement given alongside with the results $( \pm 5 \mathrm{~mm})$ comes from fluctuations with space and time of the height of the free surface. As discussed in the following sections two heights are important in our system: firstly, $H$, the height of the free surface with respect to the bottom of the mixer and, secondly, $H^{*}$, the level above the half-sphere plane.

\subsection{Shear cell}

The flow properties of the two sands at different liquid contents were characterized through an annular shear cell (Powder Flow Tester, PFT, Brookfield). Samples of $500 \mathrm{~g}$ of wet sand were prepared for each tests. Care was taken in order to standardize the procedure before and during the water addition step. Sand was dried in a ventilated oven for $4 \mathrm{~h}$ at $100^{\circ} \mathrm{C}$ and let cool at ambient temperature. The desired amount of tap water was 
then progressively added to the sample. A short stirring ( 3-4 min), carried out manually, was sufficient to ensure a proper distribution of the liquid in the sand. This procedure was based on visual inspection of the wet samples and on previous analogous experiments assisted by X-ray tomography on calcium carbonate particles (Santomaso et al., 2018). The sample was then immediately loaded in the shear cell to minimize water evaporation. The average solid fraction $v$, internal friction angle $\phi$ and cohesion $C$ were measured under application of consolidation stresses $\sigma_{\text {cons }}$ in the range 0.289 and $4.819 \mathrm{kPa}$. For an ideal material following the Mohr-Coulomb law (which is a reasonable assumption for sand), the cohesion $C$, the end-point consolidation stress $\sigma_{\text {cons }}$ and the internal friction angle $\phi$ are identified in Fig. 4 once the incipient yield locus of the material ( $\tau$ vs. $\sigma$ ) is measured. In order to quantify the effect of water addition on the mechanical behavior of the granular materials, four parameters were given particular attention: the average solid fraction $v$, the effective friction $\mu=\tan \phi$, the normalized cohesion $C / \sigma_{\text {cons }}$, and the ratio of the shear stress at incipient yield in wet and dry conditions $\tau_{\text {wet }} / \tau_{d r y}$. These parameters were studied as a function of sand type, water amount and consolidation stress. In order to estimate the uncertainty of the measurements, 3 repetitions were made for both sands and for two water amounts (dry and $w=10 \%$ ), and the standard deviation of the data was used as measure of the uncertainty.

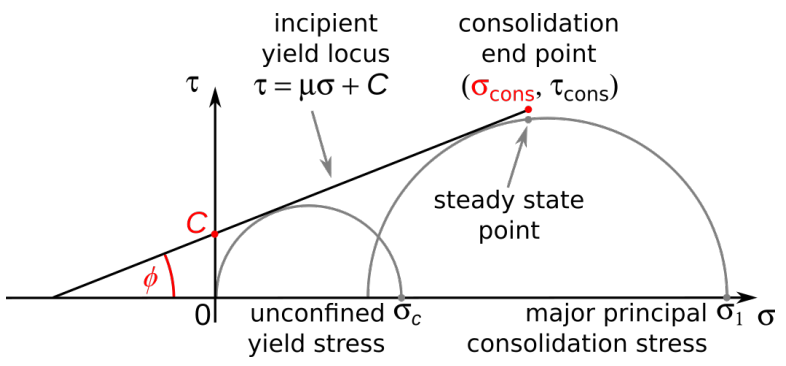

Figure 4: Mohr-Coulomb representation of the material parameters obtained with the shear cell.

\subsection{Experimental procedure}

In this work, we studied the effect of (i) the depth of immersion of the probe, (ii) the velocity and (iii) the amount of liquid added on the bending moment on the shaft of the probe resulting from the drag induced by the granular material. In a typical test, dry sand was loaded into the mixer at the beginning. Water was added in successive steps, up to saturation for the fine sand, and up to $w \approx 13 \%$ for the coarse sand ( $w$ is the mass ratio of the water to the sand). For the fine sand (FS), saturation was observed around $w \approx 30 \%$, when a water film started to form at the bed surface. As it will be discussed in the following, for coarse sand we stopped at $w \approx 13 \%$ due to homogeneity issues. For each water amount, the material was first mixed with the liquid at the highest velocity until the mixture appeared to be homogeneous (which took nearly 5 minutes). Homogenization was made with the highest level. Different levels were obtained by partially voiding the vessel. The filling level was set by controlling the mass in the mixer. For the tests with water, three filling levels were studied, corresponding to 600,800 and $1000 \mathrm{~kg}$ of sand. For FS, handling the material close to saturation revealed to be very complex, particularly with respect to partially voiding the mixer for varying the level. Above $w \approx 25 \%$, it was therefore chosen to study only one filling level (corresponding to $600 \mathrm{~kg}$ of sand). Close to saturation, it was needed to seal the mixer discharge gate to avoid water leakage. In such cases it was therefore not possible to partially empty the mixer in order to vary the filling level. For this reason, handling FS material close to saturation was very difficult due to the large quantity of material that had to be removed manually from the mixer. Therefore, above $w \approx 25 \%$, it was chosen to study only one filling level (corresponding to $600 \mathrm{~kg}$ of sand). For each level, 6 angular velocities of the mixer were tested, and the signal was acquired for more than one minute for each case. The level was manually measured every time.

As discussed in the following sections two heights are important in our system: firstly, $H$, the height of the free surface with respect to the bottom of the mixer and, secondly, $H^{*}$, the level above the half-sphere plane. For each water addition the effective water content was measured at the beginning and the end of the tests, by drying and weighing material samples. After the tests for one water amount were completed, the material extracted for varying the level was loaded again into the mixer and new water was added to increase the water content for the further tests.

\section{Results}

Figure 5 displays the effect of the mass of sand and velocity on the moment measured by the probe for the materials in the dry state. It is important to note that while Fontainebleau sand was characterized by a negligible initial water content, the different process used for drying the Teillé sand ended with a water content around $0.5 \%$. However, while not perfectly dry, it was verified that the sand was still free flowing at this value 
of water amount. Fig. 5 is given as a reference for the dry case. We can see that the moment is independent of velocity in the range considered, and it increases when the mass of the solids increases. FS is characterized by slightly larger values of the moment; this is not necessarily due to a direct effect of particle size. As it will be clearer in the following, this is probably more related to a slight difference in the solid fraction of the medium.

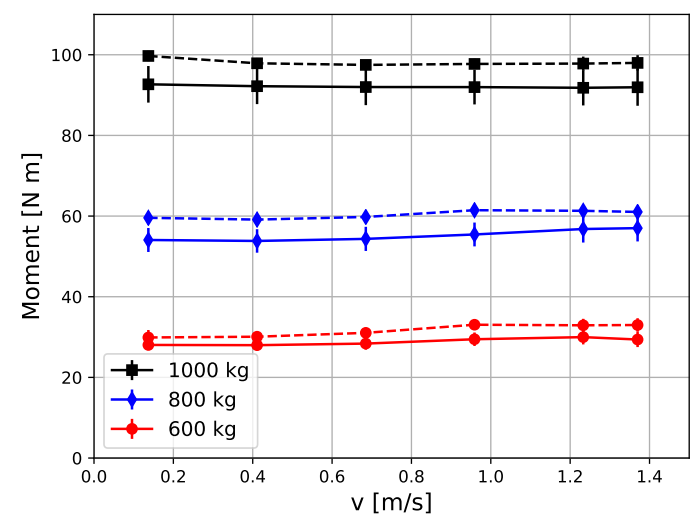

Figure 5: Average moment recorded by the probe as a function of probe velocity and amount of sand in the mixer for the "dry" materials, for Fontainebleau sand (dashed lines, $w<0.01 \%$ ) and Teillé sand (solid lines, $w=0.46 \%$ ).

Fig. 6 displays an example of the effect of the mass of sand and velocity on the moment measured in a wet configuration (around 4\%). When adding water to the system, the difference between the two materials increases; rate-independence is also confirmed, as soon as the material remains unsaturated. For example, for water amounts above the saturation value (not shown here), the moment may decrease with velocity given that at high velocity the mixing action partially suspends the sand, while at low velocity sand sediments, and thus requires a higher effort to be displaced. For water amounts above $8 \%$, Teillé sand is not well homogenized by the mixing process and the bed is formed by unstable aggregates. Above 13\%, the coarse nature of the grains induces partial percolation of water which migrates towards the bottom of the vessel. For this reason, TS data stop at $w \approx 13 \%$, while for FS it was possible to reach the saturation without observing heterogeneities in the mixture. However, it has to be noted that, for TS, the moment is quite independent of the velocity even in presence of such heterogeneities.

In Figure 7, the average moment measured at an intermediate velocity value is plotted with respect to the effective water content. The effect of the initial mass of

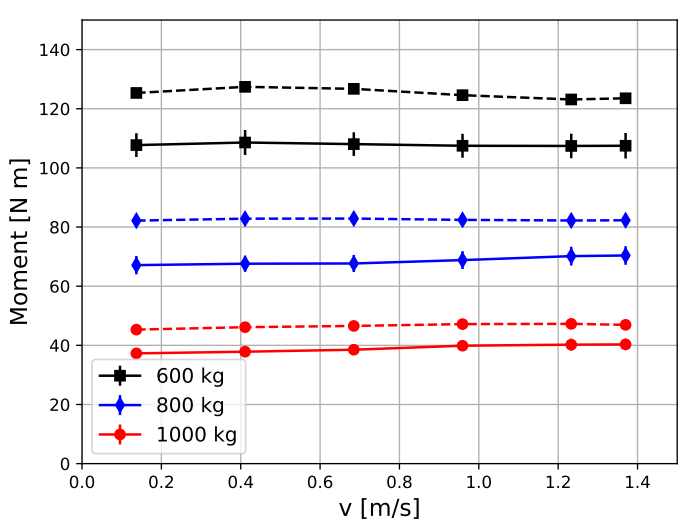

Figure 6: Average moment recorded by the probe as a function of probe velocity and amount of sand in the mixer for wet materials with a water amount around 4\%, for Fontainebleau (dashed lines) and Teillé sand (solid lines).

sand appears to be similar between wet and dry materials. Then, water addition clearly increases the resistance to the motion of the probe by the material, and we can see how for TS (corresponding to the phenomenology described above), the moment drops down around $w \approx 10 \%$, while for FS it increases up to $w \approx 20 \%$, after which it decreases. Given that for FS, the decrease in the moment is close to saturation, it could be ascribed, for example, to lubrication of interparticle contacts by the surrounding fluid. On the other hand, for TS a sudden drop of the moment is obtained for lower values of $w$, which seems to be related to the developement of a fractured, unstable, bed which was highlighted previously.

\section{Discussion}

\subsection{Phenomenology}

As discussed in the introduction, previous literature has already focused on the force acting on an object moving through a dry granular material. To summarize, the main known features of dry granular drag are that the force is independent of velocity, independent (or weakly dependent) of particle size, but dependent on depth and on object size (and shape). In our case, the independence of drag on velocity is confirmed for dry as well as for wet cases, for all the water amounts tested. This suggests that the frictional, plastic nature of granular materials may remain relevant even for high water amounts. This idea is qualitatively supported by 


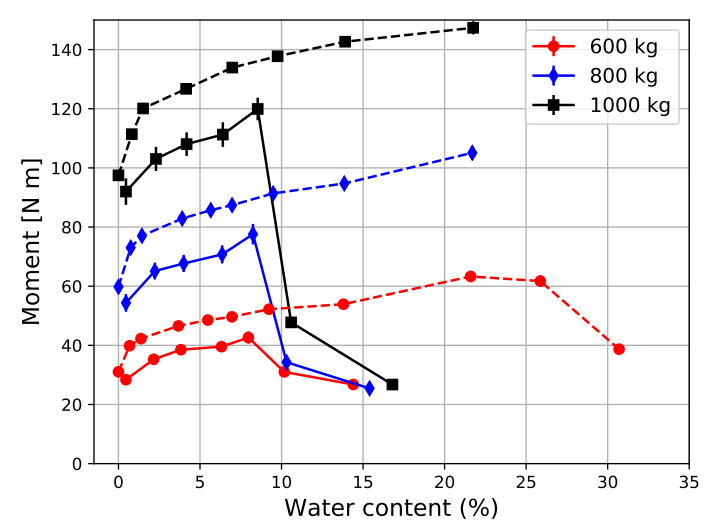

Figure 7: Average moment recorded by the probe as a function of water amount and amount of sand in the mixer, for Fontainebleau (dashed lines) and Teillé sand (solid lines). The data correspond to a probe velocity of $0.68 \mathrm{~m} / \mathrm{s}$.

the increase of the measured moment with the initial mass of material, which appears to be similar for wet and dry cases (apart from TS for $w>10 \%$, which will be discussed below). From Figure 7, it is clear that the addition of water into the system yields an increase in the resistance to the motion of the probe, for both TS and FS. It is tempting to directly ascribe this effect to the cohesion induced by water, by thinking that cohesion increases the stress which is needed to shear the material. However, as pointed out by Santomaso et al. (2018), the addition of water has also a lubricating effect: if the cohesion increases, internal friction may decrease. Apart from these effects, due to the free surface nature of the flow configuration, another important phenomenon was observed, which is the dilation of the bed with the addition of water. This effect is shown in Figure 8 , in which it is clear that cohesion stabilizes loose configurations, and the height of material in the vessel increases when water is added, reaching a value which can be up to 1.4 times the initial level. This increase is observed until a water amount around $w=10 \%$, after which the level decreases. The addition of water implies, for the same solid fraction, an increase of the mass of the material above the probe, and so, the simple fact of adding a liquid could yield an increase in the measured moment due to the extra mass. The dilation induced by cohesion, though reducing the overall bulk density, acts in the same direction.

Given that the position of the probe is constant with respect to the bottom of the vessel, an increase of the level due to dilation means that a higher weight of material interferes with the probe (and this even if the av-

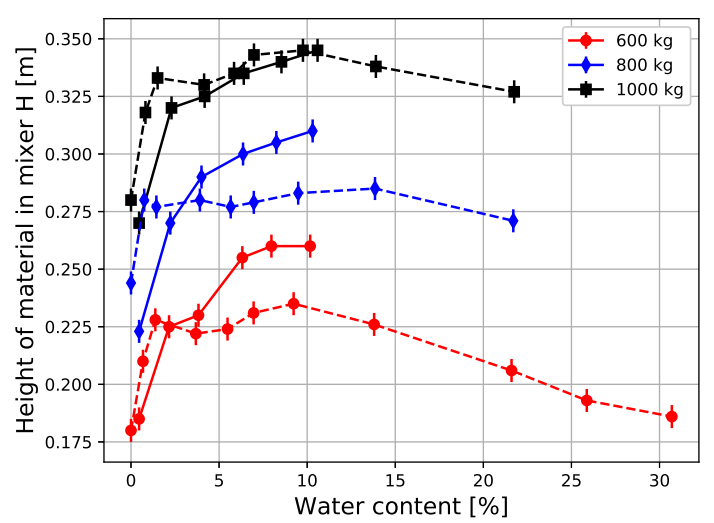

Figure 8: Evolution of the height of material in the mixer as a function of water amount and amount of sand in the mixer. Dashed lines correspond to FS and solid lines to TS. Water induces dilatancy up to $w=10 \%$ due to the free surface nature of the flow configuration.

erage solid fraction is lower) and will contribute to increasing the measured moment. Therefore four phenomena induced by the liquid addition may contribute to change the resistance to motion provided by the material: cohesion (due to capillary forces), dilatancy (induced by cohesion), extra liquid mass, and internal friction (modulated by contacts lubrication). In the following we try to analyse the data presented in Section 3, with the aim of understanding the effect of these phenomena on drag.

\subsection{Scaling of the moment with $H$ for dry materials}

In order to evaluate the effect of dilatancy, the easiest way is to compare the moment measured in a wet configuration with an equivalent dry configuration characterized by the same level and solid fraction. While we expect from literature that the dependence of forces will be linear on solid fraction, the effect of the level is system-specific (its scaling depends on the probe size and shape). For this reason, a series of measurements on FS was devoted to characterize the dependence of the measured bending moment on the level, for the dry case. In Figure 9, in order to find a scaling, we plot the moment $M$ rescaled by $\rho g$, where $\rho$ is the average bulk density, estimated from the mass and the measured level, as a function of the level (here taken with respect to the half plane of the spherical part of the probe, and noted as $\left.H^{*}\right)$. A fitting law can be extracted from these data, characterising the scaling of the moment on solid fraction and depth. Due to the discontinuity related to the passage from the cylinder to the sphere, we expect that a 
different fitting function should be used when the material is only partially covering the sphere $\left(H^{*}<0.045 \mathrm{~m}\right.$ in Figure 9). In this case, given that wet mixtures were always obtained for levels above the sphere, we fitted only the part of the curve above $H^{*}=0.045 \mathrm{~mm}$.

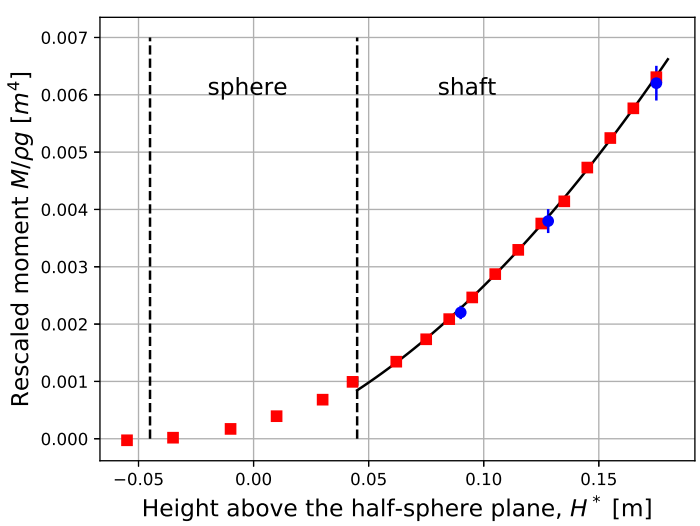

Figure 9: Average moment recorded by the probe for dry materials (squares: FS, circles: TS), rescaled by $\rho g$, as a function of the level above the half-sphere plane $H^{*}$.

As it is visible in Fig. 9, our data is well fitted by the relation:

$$
\frac{M}{\rho g}=f_{d r y}\left(H^{*}\right)=\alpha H^{* 2}+\beta H^{*}+\delta
$$

where $\alpha=1.21 \times 10^{-1}\left[\mathrm{~m}^{2}\right], \beta=1.55 \times 10^{-2}\left[\mathrm{~m}^{3}\right]$, $\delta=-9.98 \times 10^{-5}\left[\mathrm{~m}^{4}\right]$. While the fit was obtained on FS, it is surprising to see that the "dry" points of TS fall on the same curve; this may be related to the fact that both TS and FS are rounded siliceous sands, and this supports the idea of a similar macroscopic response. For this reason, we will use the same functional form for the two sands in the following. The form of Eq. 1 is consistent with the work of Albert et al. (1999, 2001a). Note also, that the evolution of the force (and consequently the moment) versus the depth of the probe is far from being trivial due to the discrete nature of sand (Albert et al., 2001a).

\subsection{Extra mass effects vs mechanical cohesion}

From the previous discussion, it is clear that a mass increase in the mixer (due to an addition of solids or liquid), or a level raise due to capillarity-induced dilation, may augment the mass above the probe and therefore increase the resistance to motion of the intruder. It can be interesting to try to separate these "extra mass" effects from the cohesion itself, which, despite having the same micromechanical origin as dilatancy (i.e. capillary forces), can be seen as a separate effect whose importance can depend on the flow configuration. A way to separate the two phenomena is to study the ratio between the moment measured in wet and dry conditions, and to split it by means of the obtained scaling for dry materials:

$$
\frac{M_{w e t}}{M_{d r y}}=\tilde{M}_{a m} \tilde{M}_{c o h}=\frac{\rho_{w e t} g f_{d r y}\left(H_{w e t}\right)}{M_{d r y}} \frac{M_{w e t}}{\rho_{w e t} g f_{d r y}\left(H_{w e t}\right)},
$$

where the scaling for dry materials is introduced to mimick the extra mass effect provided by an increase in the mass of the solids or the liquid, or by dilation, and where $\rho_{\text {wet }}$ is the average bulk density estimated from the level and the mass of materials, considering also the addition of water. Therefore, the first factor in the RHS of Eq. 2, $\tilde{M}_{a m}$, can be used as a measure of the importance of the extra mass effects, while the second factor, $\tilde{M}_{c o h}$, is meant to characterize separately the effect of mechanical cohesion. The results of the normalization is displayed in Figure 10, where error bars are computed by propagation of the uncertainties of level and moment measurements. The first observation is that at least for low values of the water content, $\tilde{M}_{c o h}$ is close to 1 , while $\tilde{M}_{a m}$ increases significantly with the water amount. This suggests that for low water contents, for both sands the main mechanism affecting drag is provided by the extra mass effects, and therefore the drag force is determined by the effective depth of immersion of the probe. For a given mass of solids, the strongest effect of the addition of water on the moment recorded by the probe seems to be related to the variation in level due to cohesioninduced dilation.

However, if we look in more detail at the materialdependent behavior, the two sands employed behave differently. From Fig. 10a, it is clear that the contribution of extra mass effects is higher for TS than for FS, which means that the dilation induced by the liquid addition is more important for the coarse sand. This is clearly related to the variation in level after water addition, which is stronger for TS than for FS (Fig. 8). Also, the clear decrease of the level observed for the lowest filling of FS results in a decrease of $\tilde{M}_{a m}$ with the water content, for $w>10 \%$. With respect to Fig. 10b, for Teillé sand, the addition of water corresponds to a slight decrease in $\tilde{M}_{c o h}$, which may be the signature of lubrication. Teille sand is the coarser sand used in this work, so it is reasonable to think that lubrication may interfere with cohesion for it. For $w \approx 10 \%$, where 

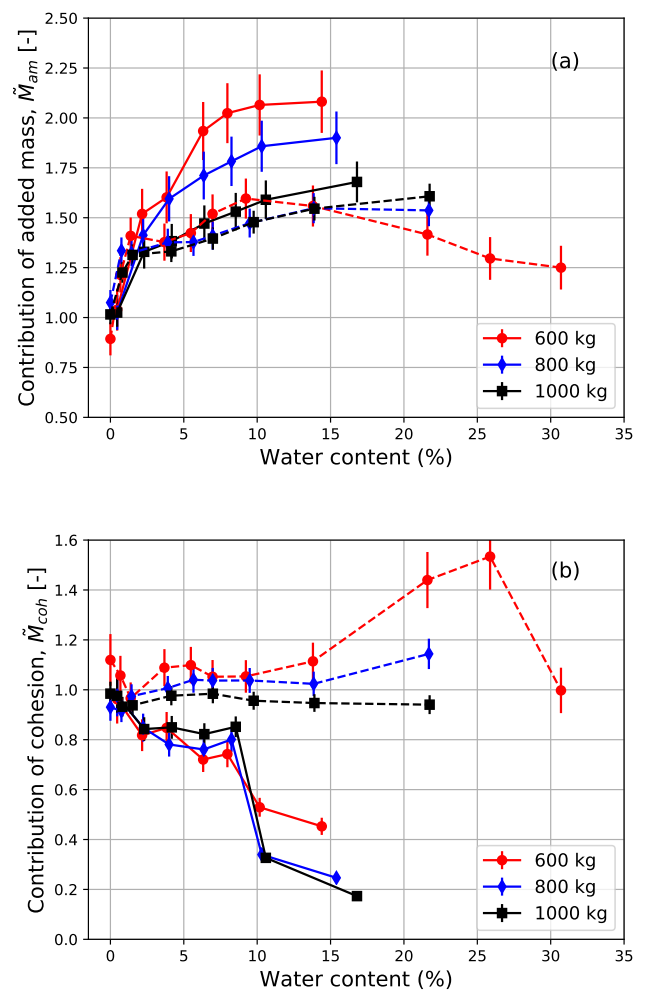

Figure 10: Contribution of (a) the extra mass effect induced by addition of solids, liquid and by dilation and (b) mechanical cohesion on the average moment recorded by the probe as a function of water amount and mass of sand in the mixer, for Fontainebleau (dashed lines) and Teillé sand (solid lines). Same data as in Fig. 7.

the unrescaled data (see Fig. 7) displayed an abrupt decrease of the moment, $\tilde{M}_{c o h}$ decreases as well, and the points corresponding to the different initial levels do not superpose, which means that the scaling on depth based on dry data does not hold. As it was partially discussed previously, for water amounts larger than $10 \%$, TS starts to be very heterogeneous and forms lumps which are not easily broken by the mixing process. The material is very dilated and fractured, and the probe easily penetrates through this fractured bed. This is probably the phenomenon originating the progressive failure of the scaling obtained for dry data, when $w \geq 10 \%$. For Fontainebleau sand, the picture is clearly different. When increasing the water amount, the effect of the initial mass of the solids becomes increasingly important for this fine sand. $\tilde{M}_{c o h}$ is larger the lower the mass of the solids, and the difference between the curves increases. This means that the scaling of the moment with depth obtained for dry materials progressively fails as cohesion becomes more important. The order of the curves in Fig. 10b is also important. For the highest initial level, $\tilde{M}_{c o h}$ is nearly constant with respect to $w$, and slightly lower than 1 . This means that the moment measured by the probe is close to the one corresponding to a dry bed. When the initial level is lower, the moment exceeds the dry reference value by up to $50 \%$. To summarize, Fig. 10a and Fig. 10b show that the socalled extra mass effects were generally very important in our experiments, apart from the lowest filling level of the fine sand, where for high water amounts mechanical cohesion played a major role. In other words, while the effect of cohesion is important only close to the free surface, the effect of water addition is felt also by the deep layers through the variation in level induced by dilation. This behavior is due to the fact that the importance of cohesion must be considered relatively to body forces. In our case, body forces increase as we move deep inside the bed; moreover, due to the shape of the probe, the frictional nature of the drag and the nature of the quantity measured, the total moment measured does not represent equally all the layers of material touching the immersed part of the probe, but gives more importance to the deepest layers. It is therefore reasonable to think that there is a position in the bed below which cohesive forces are overcome by the mass of the material: this yields the fact that for $1000 \mathrm{~kg}$ of sand, the moment measured follows the scaling obtained for the dry material. If the probe is sensing less deeper layers, it is allowed to register the effect of cohesion. We can ascribe the excess of moment with respect to the reference scaling to an increase in cohesion. This marks an important difference with respect to Teillé sand, for which the effect of cohesion was not visible. This difference is clearly related to the particle size, which drives the importance of capillary forces with respect to body forces. As it was stated in the Introduction, a dimensionless number called Bond number can be used to quantify the sensitivity of a granular material to capillary forces. In our case, $B o$, computed with the median diameter, is respectively 0.17 and 0.022 for TS and FS sands. Even if Bond number does not consider the contribution of the confinement stress, and is therefore more relevant close to the bed surface, it gives an indirect proof that for both sands, capillarity can influence the material behavior, but that FS is more sensitive. In addition, one should consider that TS has a broader size distribution than FS, and given that the finest particles in the distribution $\left(d<d_{10}\right)$ have particle sizes comparable to FS, this may increase the sensitivity of FS to the water addition. 


\subsection{Shear cell results}

In order to understand the origin of the different mechanical behaviors observed with the two types of sand, we discuss in this section the results obtained with the shear cell described in Sec. 2.5. In particular, in Fig. 11 we plot different parameters obtained from the shear cell as a function of water content for the two types of sand and different consolidation stresses. An increase of the consolidation stress mimics an increase of the level in the mixer: in the latter, the material is sheared under its own load and so we can assume that the consolidation stress increases with depth. Given that the probe is fixed, this means that the consolidation stress near the probe end increases with the level of the material. The three values of consolidation stress used in the shear cell are of the same order of magnitude of the hydrostatic pressure near the end of the probe for the different levels in the mixer. However, as it was highlighted in the previous sections, the level in the mixer varied significantly upon the addition of water, while in the shear cell the consolidation stress was kept the same for the sake of simplicity while varying the water content. The shear cell results are not meant to provide a punctual comparison, but were done to obtain an independent measurement of the effect of water addition on the forces needed to shear the material.

The average solid fraction of the materials at incipient yield is displayed in Fig. 11a. TS has generally a higher solid fraction than FS, probably because of the highest polydispersity. This feature causes the formation a more compact packing. It is interesting to note that $v$ decreases when adding water up to $w=5 \%$ and then remains nearly stable. The material loosens when adding water, and this reflects the behavior observed in the mixer (Fig. 8). However, the minimum of solid fraction is obtained for lower values of the water amount than the maximum of the level in the mixer, and above $10 \%$ the level in the mixer decreases with $w$, which is not mirrored by the solid fraction in the cell. This is probably due to the different geometry: in the shear cell, the material can partially expand due to the stresscontrolled configuration, nervertheless it remains a fully confined setup. On the other hand, the mixer is a free surface configuration in which dilation is probably less disturbed. In addition, the bulk solid fraction can be assumed as uniform in the shear cell while it will probably depend on depth in the mixer.

In Fig. $11 \mathrm{~b}$, the effective friction $\mu$ (the slope of the incipient yield locus) is displayed. TS is characterized by higher values of the friction coefficient, which can be related to a higher level of interlocking between the particles due to the higher solid fraction. It is evident that the addition of water decreases $\mu$ at least until $w<5 \%$. An increase of $\mu$ is then apparent for $w>10 \%$. With respect to effective friction, the two types of sand respond slightly differently to the addition of water, with the finer (FS) displaying a larger variation of $\mu$. A decrease of friction with $w$ for $w<5 \%$ was already discussed by Santomaso et al. (2018), who postulated that the micromechanical origin of such phenomenon could be related to the lubrication of interparticle contacts by the wetting liquid. While this is confirmed by the present data, we also observe a non-monotonic variation of $\mu$ for higher water contents which would require further investigation.

Fig. 11c displays the evolution of cohesion parameter $C$, normalized by the consolidation stress $\sigma_{\text {cons }}$, with the water content. As one may expect, cohesion generally increases when the liquid is added to the granular material, and this increase is nonlinear, with a larger variation between $w=0$ and 2\%. The coarse sand appears to be less sensitive to cohesion $\left(C / \sigma_{\text {cons }}\right.$ for FS is roughly $4 / 3$ of $C / \sigma_{\text {cons }}$ for TS), which is the expected effect of particle size, and the importance of cohesion decreases when increasing the consolidation stress. This behavior is analogous to what was observed in the mixer (Fig. 10), where the effect of cohesion on the measured moment decreases with the filling level of the mixer. Together, Figs. $11 \mathrm{~b}$ and $11 \mathrm{c}$ show the importance of both internal friction and cohesion phenomena for the two types of sand studied in this work.

In order to evaluate the effect of water addition on the global behavior of the mixture, we plot in Fig. 11d, the ratio of the shear stress at incipient yield in wet conditions to the one in the dry case. This quantity is related to the normalized moment shown in Fig. 10, because it compares the effort needed to shear the material in wet and dry conditions. From the figure it is evident that $\tau_{\text {wet }} / \tau_{d r y}$ increases with the water addition: this means that the decrease in the slope of the yield locus is always compensated and exceeded by an increase in cohesion. This is different from drag data, in which the coarser TS displayed a decrease of the normalized moment with $w$, and therefore a global balance in favour of internal friction, modulated by contact lubrication. The opposite variation of $\tau_{w e t} / \tau_{d r y}$ in the shear cell and $\tilde{M}$ in the mixer have probably to be ascribed to the different flow configuration, and particularly to the more pronounced confinement provided by the shear cell, as it was discussed above with respect to solid fraction. However, apart from the variation, it has to be noted that $\tau_{\text {wet }} / \tau_{d r y}$ for TS seems to be insensitive to the consolidation stress; this peculiar behavior finds a correspondence in Fig. 10 and can be seen as a signature of the 

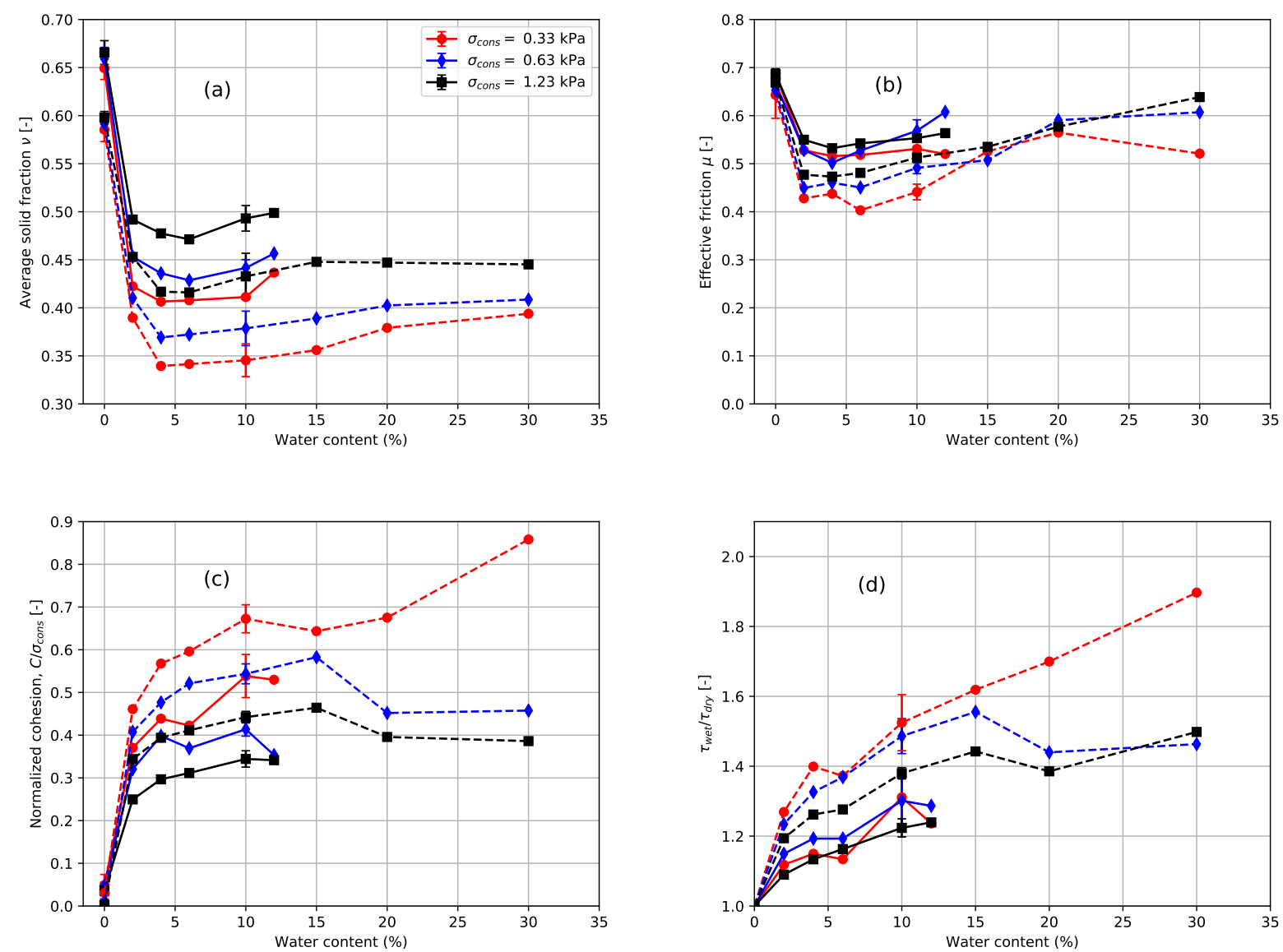

Figure 11: Effect of sand type, water content and consolidation stress on bulk material parameters estimated with a shear cell : (a) average solid fraction $v$ at incipient yield, (b) effective friction coefficient $\mu=\tan \phi$, (c) normalized cohesion $C / \sigma_{\text {cons }}$, and (d) ratio of the shear stress at incipient yield in wet and dry conditions $\tau_{w e t} / \tau_{d r y}$.

reduced importance of cohesion for the coarser sand. On the other hand, for the finer sand (FS), the effect of water visible in Fig. 11d is stronger and the curves are sensitive to the consolidation stress $\sigma_{\text {cons }}$. Even in this case, the shear cell provides an overestimation of the effect of water with respect to the results obtained in the mixer, but it is important to notice that the order of the curves is the same that in Fig. 10, and the curve corresponding to the highest consolidation stress is close to the curves obtained for TS. The origin of the increase in the yield stress upon the addition of water in conditions for which we would normally suppose that the liquid is negligible (coarse particles, high consolidation stress) needs further investigation. Still, shear cell results confirm that the drag force on an object moving through a wet granular material is a complex phenomenon which involves the effect of cohesion, dilatancy and internal friction. These three phenomena may all be relevant at the same time, as the present results suggest.

\section{Conclusions}

The present paper reports an experimental study of the drag force applied on an object moving through a wet granular medium with the aim to compare our results to those obtained with the well-known dry case. We used both fine (Fontainebleau sand, $d_{50} \approx 250 \mu \mathrm{m}$ ) and coarse (Teillé sand, $d_{50} \approx 690 \mu \mathrm{m}$ ) sands. First, the moment of the drag force is found to be independent on the velocity of the probe and to increase with the amount of granular medium, similar to what has been observed for dry granular materials. Yet, strong differences between the dry and wet cases exist, consequences of (i) cohesion due to capillary forces, (ii) pos- 
sible contact lubrication and (iii) dilation induced by cohesion. We have shown that, among the three aforementioned mechanisms, the effect of dilation is the most important, at least in our experimental configuration. The effect of cohesion at the system scale depends on the relative influence of the cohesive forces with respect to the grains' weight. This implies that, for equal material densities, a coarse sand is less sensitive to cohesion than a fine one. Consequently coarse grains may experience a significant contact lubrication. At important water contents, the granular system may become strongly heterogeneous.

We also carried out shear cell experiments to obtain an independent measurement of the effect of water content on the stress required to shear the granular media used in the industry-scale mixer. Our results confirm that both contact lubrication (which prevails at low water content) and cohesion influence the mechanical response of wet granular systems. They also shed light on the importance of the geometry of the system which by its characteristics (e.g. the existence of a free surface) influences the dilation of the system and thus its behavior.

\section{Acknowledgments}

We thank D. Hamon, A. Cothenet, F. Buisson for the help with the sample preparation and mixer operation and J. M. Paul for the development of the sensor.

\section{References}

Albert, I., Sample, J. G., Morss, A. J., Rajagopalan, S., Barabási, A.L., Schiffer, P., Nov 2001a. Granular drag on a discrete object: Shape effects on jamming. Phys. Rev. E 64, 061303.

Albert, I., Tegzes, P., Albert, R., Sample, J. G., Barabási, A. L., Vicsek, T., Kahng, B., Schiffer, P., Aug 2001b. Stick-slip fluctuations in granular drag. Phys. Rev. E 64, 031307.

Albert, R., Pfeifer, M. A., Barabási, A.-L., Schiffer, P., Jan 1999. Slow drag in a granular medium. Phys. Rev. Lett. 82, 205-208.

Artoni, R., Santomaso, A. C., Gabrieli, F., Tono, D., Cola, S., Mar 2013. Collapse of quasi-two-dimensional wet granular columns. Phys. Rev. E 87, 032205.

Badetti, M., Fall, A., Hautemayou, D., Chevoir, F., Aimedieu, P., Rodts, S., Roux, J.-N., Sep. 2018. Rheology and microstructure of unsaturated wet granular materials: Experiments and simulations. Journal of Rheology 62, 1175-1186.

Bouwman, A., Henstra, M., Hegge, J., Zhang, Z., Ingram, A., Seville, J., Frijlink, H., 2005. The relation between granule size, granule stickiness, and torque in the high-shear granulation process. Pharmaceutical research 22 (2), 270-275.

Cavinato, M., Artoni, R., Bresciani, M., Canu, P., Santomaso, A. C., 2013. Scale-up effects on flow patterns in the high shear mixing of cohesive powders. Chemical Engineering Science 102, 1-9.

Cavinato, M., Bresciani, M., Machin, M., Bellazzi, G., Canu, P., Santomaso, A. C., 2010. Formulation design for optimal high-shear wet granulation using on-line torque measurements. International journal of pharmaceutics 387 (1-2), 48-55.
Cazacliu, B., 2008. In-mixer measurements for describing mixture evolution during concrete mixing. chemical engineering research and design 86 (12), 1423-1433.

Cazacliu, B., Roquet, N., 2009. Concrete mixing kinetics by means of power measurement. Cement and Concrete Research 39 (3), 182194.

Chehata, D., Zenit, R., Wassgren, C. R., 2003. Dense granular flow around an immersed cylinder. Physics of Fluids 15 (6), 1622-1631.

Chitu, T. M., Oulahna, D., Hemati, M., 2011. Wet granulation in laboratory scale high shear mixers: Effect of binder properties. Powder technology 206 (1-2), 25-33.

Collet, R., Oulahna, D., Ryck, A. D., Jezequel, P., Martin, M., 2011. Mixing of a wet granular medium: Influence of the liquid addition method. Powder Technology 208 (2), 367 - 371, special Issue: Papers presented to the Symposium STPMF 2009, Science and Technology of Powders and Sintered Materials.

Collet, R., Oulahna, D., Ryck, A. D., Jezequel, P. H., Martin, M., 2010. Mixing of a wet granular medium: Effect of the particle size, the liquid and the granular compacity on the intensity consumption. Chemical Engineering Journal 164 (2), 299 - 304, pharmaceutical Granulation and Processing.

Gabrieli, F., Artoni, R., Santomaso, A., Cola, S., 2013. Discrete particle simulations and experiments on the collapse of wet granular columns. Physics of Fluids 25 (10), 103303.

Guillard, F., Forterre, Y., Pouliquen, O., Mar 2013. Depthindependent drag force induced by stirring in granular media. Phys. Rev. Lett. 110, 138303.

Jørgensen, A. C., Luukkonen, P., Rantanen, J., Schæfer, T., Juppo, A. M., Yliruusi, J., 2004. Comparison of torque measurements and near-infrared spectroscopy in characterization of a wet granulation process. Journal of pharmaceutical sciences 93 (9), 2232-2243.

Kumar, S., Anki Reddy, K., Takada, S., Hayakawa, H., Dec. 2017. Scaling law of the drag force in dense granular media. ArXiv eprints.

Leuenberger, H., 1982. Granulation, new techniques. Pharm Acta Helv 57, 72-82

Liao, C.-C., Hsiau, S.-S., 2010. Experimental analysis of dynamic properties in wet sheared granular matter. Powder Technology 197 (3), $222-229$.

Louati, H., Oulahna, D., de Ryck, A., 2015. Apparent friction and cohesion of a partially wet granular material in steady-state shear. Powder Technology 278, 65 - 71 .

Louati, H., Oulahna, D., de Ryck, A., 2017. Effect of the particle size and the liquid content on the shear behaviour of wet granular material. Powder Technology 315, 398 - 409.

Santomaso, A. C., Volpato, S., Gabrieli, F., 2018. Collapse and runout of granular columns in pendular state. Physics of Fluids 30 (6), 063301.

Schindelin, J., Arganda-Carreras, I., Frise, E., Kaynig, V., Longair, M., Pietzsch, T., Preibisch, S., Rueden, C., Saalfeld, S., Schmid, B., Tinevez, J.-Y., White, D. J., Hartenstein, V., Eliceiri, K., Tomancak, P., Cardona, A., 2012. Fiji: an open-source platform for biological-image analysis. Nat Meth 9 (7), 676-682.

Seguin, A., Bertho, Y., Gondret, P., Crassous, J., Jul 2011. Dense granular flow around a penetrating object: Experiment and hydrodynamic model. Phys. Rev. Lett. 107, 048001.

Wassgren, C. R., Cordova, J. A., Zenit, R., Karion, A., 2003. Dilute granular flow around an immersed cylinder. Physics of Fluids 15 (11), 3318-3330.

Wieghardt, K., 1974. Forces in granular flow. Mechanics Research Communications 1 (1), $3-7$.

Wieghardt, K., 1975. Experiments in granular flow. Annual Review of Fluid Mechanics 7 (1), 89-114. 